Acta Crystallographica Section E

\section{Structure Reports}

Online

ISSN 1600-5368

\section{4-Amino-3-[(4-methoxyphenyl)amino- methyl]-1H-1,2,4-triazole-5(4H)-thione}

\author{
Hoong-Kun Fun, ${ }^{a *} \ddagger$ Chin Sing Yeap, ${ }^{a} \S$ Shridhar Malladi, \\ Mahesh Padaki $^{b}$ and Arun M. Isloor ${ }^{b}$
}

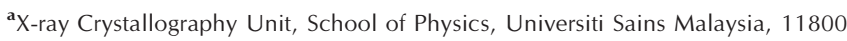
USM, Penang, Malaysia, and ${ }^{\mathbf{b}}$ Department of Chemistry, National Institute of Technology-Karnataka, Surathkal, Mangalore 575 025, India

Correspondence e-mail: hkfun@usm.my

Received 17 August 2009; accepted 17 August 2009

Key indicators: single-crystal X-ray study; $T=100 \mathrm{~K}$; mean $\sigma(\mathrm{C}-\mathrm{C})=0.003 \AA$; $R$ factor $=0.031 ; w R$ factor $=0.068 ;$ data-to-parameter ratio $=13.8$.

The molecule of the title compound, $\mathrm{C}_{10} \mathrm{H}_{13} \mathrm{~N}_{5} \mathrm{OS}$, is approximately planar, the dihedral angle between the triazole and benzene rings being $4.53(10)^{\circ}$. The amino group adopts a pyramidal configuration. In the crystal structure, molecules are linked into two-dimensional networks parallel to (001) by intermolecular $\mathrm{N}-\mathrm{H} \cdots \mathrm{S}$ and $\mathrm{N}-\mathrm{H} \cdots \mathrm{N}$ hydrogen bonds. In addition, an S...S short contact of 3.3435 (7) $\AA$ is observed.

\section{Related literature}

For the pharmacological applications of 1,2,4-triazole derivatives, see: Amir et al. (2008); Isloor et al. (2009); Krzysztof et al. (2008); Kuş et al. (2008); Padmavathi et al. (2008). For the preparation, see: Holla \& Udupa (1992). For related structures, see: Fun et al. $(2009 a, b)$. For the stability of the temperature controller used for data collection, see: Cosier \& Glazer (1986).

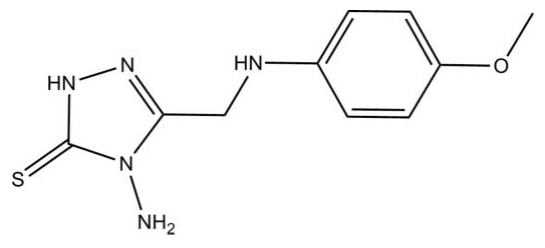

\section{Experimental}

Crystal data

$\mathrm{C}_{10} \mathrm{H}_{13} \mathrm{~N}_{5} \mathrm{OS}$

$M_{r}=251.31$

Monoclinic, $C 2$

$a=11.5142$ (2) $\AA$

$b=5.8804$ (1) $\AA$

$c=16.6891$ (3) $\AA$

$\beta=95.292$ (1)
Data collection

Bruker SMART APEXII CCD area-detector diffractometer

Absorption correction: multi-scan (SADABS; Bruker, 2005)

$T_{\min }=0.913, T_{\max }=0.994$

Refinement

$R\left[F^{2}>2 \sigma\left(F^{2}\right)\right]=0.031$

$w R\left(F^{2}\right)=0.068$

$S=1.04$

2365 reflections

171 parameters

1 restraint
5827 measured reflections 2365 independent reflections 2126 reflections with $I>2 \sigma(I)$ $R_{\text {int }}=0.024$

$\mathrm{H}$ atoms treated by a mixture of independent and constrained refinement

$\Delta \rho_{\max }=0.33{\mathrm{e} \AA^{-3}}^{-3}$

$\Delta \rho_{\min }=-0.26{\mathrm{e} \AA^{-3}}^{-3}$

Absolute structure: Flack (1983), 588 Friedel pairs

Flack parameter: $0.03(7)$

Table 1

Hydrogen-bond geometry $\left(\AA{ }^{\circ}\right)$.

\begin{tabular}{lllll}
\hline$D-\mathrm{H} \cdots A$ & $D-\mathrm{H}$ & $\mathrm{H} \cdots A$ & $D \cdots A$ & $D-\mathrm{H} \cdots A$ \\
\hline $\mathrm{N} 2-\mathrm{H} 1 N 2 \cdots \mathrm{S} 1^{\mathrm{i}}$ & $0.84(2)$ & $2.55(2)$ & $3.3672(18)$ & $164(2)$ \\
$\mathrm{N} 4-\mathrm{H} 1 N 4 \cdots \mathrm{N} 5^{\mathrm{ii}}$ & $0.82(3)$ & $2.60(3)$ & $3.410(2)$ & $169(3)$ \\
$\mathrm{N} 5-\mathrm{H} 1 N 5 \cdots \mathrm{N} 1^{\text {iii }}$ & $0.89(2)$ & $2.48(2)$ & $3.133(2)$ & $130(2)$ \\
\hline Symmetry codes: (i) $-x+\frac{1}{2}, y-\frac{1}{2},-z ;\left(\right.$ ii) $x-\frac{1}{2}, y-\frac{1}{2}, z ;\left(\right.$ iii) $x+\frac{1}{2}, y+\frac{1}{2}, z$.
\end{tabular}

Data collection: APEX2 (Bruker, 2005); cell refinement: SAINT (Bruker, 2005); data reduction: $S A I N T$; $\operatorname{program}(\mathrm{s})$ used to solve structure: SHELXTL (Sheldrick, 2008); program(s) used to refine structure: SHELXTL; molecular graphics: SHELXTL; software used to prepare material for publication: SHELXTL and PLATON (Spek, 2009).

HKF thanks Universiti Sains Malaysia (USM) for the Research University Golden Goose grant No. 1001/PFIZIK/ 811012. CSY thanks USM for the award of a USM fellowship. AMI is grateful to the Head of the Department of Chemistry and Director, NITK, Surathkal, India, for providing research facilities.

Supplementary data and figures for this paper are available from the IUCr electronic archives (Reference: CI2889).

\title{
References
}

Amir, M., Kumar, H. \& Javed, S. A. (2008). Eur. J. Med. Chem. 43, 2056-2066. Bruker (2005). APEX2, SAINT and SADABS. Bruker AXS Inc., Madison, Wisconsin, USA.

Cosier, J. \& Glazer, A. M. (1986). J. Appl. Cryst. 19, 105-107.

Flack, H. D. (1983). Acta Cryst. A39, 876-881.

Fun, H.-K., Goh, J. H., Vijesh, A. M., Padaki, M. \& Isloor, A. M. (2009a). Acta Cryst. E65, o1918-o1919.

Fun, H.-K., Liew, W.-C., Vijesh, A. M., Padaki, M. \& Isloor, A. M. (2009b). Acta Cryst. E65, o1910-o1911.

Holla, B. S. \& Udupa, K. V. (1992). Farmaco, 47, 305-318.

Isloor, A. M., Kalluraya, B. \& Shetty, P. (2009). Eur. J. Med. Chem. 44, 3784 3787.

Krzysztof, S., Tuzimski, T., Rzymowska, J., Kazimierz, P. \& Kandefer-Szerszeń, M. (2008). Eur. J. Med. Chem. 43, 404-419.

Kuş, C., Ayhan-Kılcıgil, G., Özbey, S., Kaynak, F. B., Kaya, M., Çoban, T. \& Can-Eke, B. (2008). Bioorg. Med. Chem. 16, 4294-4303.

Padmavathi, V., Thriveni, P., Reddy, G. S. \& Deepti, D. (2008). Eur. J. Med. Chem. 43, 917-924.

Sheldrick, G. M. (2008). Acta Cryst. A64, 112-122.

Spek, A. L. (2009). Acta Cryst. D65, 148-155. 


\section{supporting information}

Acta Cryst. (2009). E65, o2213 [doi:10.1107/S1600536809032607]

\section{4-Amino-3-[(4-methoxyphenyl)aminomethyl]-1H-1,2,4-triazole-5(4H)-thione Hoong-Kun Fun, Chin Sing Yeap, Shridhar Malladi, Mahesh Padaki and Arun M. Isloor}

\section{S1. Comment}

1,2,4-triazole and its derivatives were reported to exhibit various pharmacological activities such as antimicrobial, analgesic, anti-inflammatory, anticancer and antioxidant properties (Amir et al., 2008; Krzysztof et al., 2008; Kuş et al., 2008; Padmavathi et al., 2008). A few derivatives of triazoles have exhibited antimicrobial activity (Isloor et al., 2009). Some of the present day drugs such as ribavirin (antiviral agent), rizatriptan (antimigraine agent), alprazolam (anxiolytic agent), fluconazole and itraconazole (antifungal agents) are the best examples for potent molecules possessing the triazole nucleus. The amino and mercapto groups of 1,2,4-triazoles serve as readily accessible nucleophilic centers for the preparation of N-bridged heterocycles. Keeping in view of the biological importance, we have synthesized the title compound and its crystal structure is reported here.

Bond lengths and angles in the title compound (Fig. 1) are comparable to those observed in related structures (Fun et $a l ., 2009 a, b)$. The molecule is approximately planar, with the dihedral angle between the triazole $(\mathrm{N} 1 / \mathrm{N} 2 / \mathrm{C} 1 / \mathrm{N} 3 / \mathrm{C} 2)$ and benzene rings (C4-C9) being $4.53(10)^{\circ}$.

In the crystal structure, the molecules are linked by intermolecular N2-H1N2 $\cdots \mathrm{S} 1, \mathrm{~N} 4-\mathrm{H} 1 \mathrm{~N} 4 \cdots \mathrm{N} 5$ and N5H1N5 $\cdots \mathrm{N} 1$ hydrogen bonds into a two-dimensional network parallel to $a b$ plane (Fig. 2 and Table 1). In addition, a $\mathrm{S} \cdots \mathrm{S}(1-\mathrm{x}, \mathrm{y},-\mathrm{z})$ short contact of 3.3435 (7) $\AA$ is observed.

\section{S2. Experimental}

2-[(4-Methoxyphenyl)amino]acetohydrazide (19.5 g, $0.1 \mathrm{~mol})$ was added slowly to a solution of potassium hydroxide $(8.4 \mathrm{~g}, 0.15 \mathrm{~mol})$ in ethanol $(150 \mathrm{ml})$. The resulting mixture was stirred well till a clear solution was obtained. Carbon disulfide ( $11.4 \mathrm{~g}, 0.15 \mathrm{~mol})$ was added dropwise and the contents were stirred vigorously. Further stirring was continued for $24 \mathrm{~h}$. The resulting mixture (Holla \& Udupa, 1992) was diluted with $100 \mathrm{ml}$ of ether and the precipitate formed was collected by filtration, washed with dry ether and dried at $338 \mathrm{~K}$ under vacuum. It was used for the next step without any purification.

A mixture of above synthesized potassium dithiocarbazinate (30.9 g, $0.1 \mathrm{~mol})$, hydrazine hydrate $(99 \%, 0.2 \mathrm{~mol})$ and water $(2 \mathrm{ml})$ was gently heated to boil for $30 \mathrm{~min}$. Heating was continued until the evaluation of hydrogen sulfide ceases. The reaction mixture was cooled to room temperature, diluted with water $(100 \mathrm{ml})$ and acidified with $2 \mathrm{~N} \mathrm{HCl}$. The solid mass that separated was collected by filtration, washed with water and dried. Recrystallization was done from ethanol (yield: 15.1 g, 67.7\%; m.p. 484-486 K).

\section{S3. Refinement}

$\mathrm{N}$-bound $\mathrm{H}$ atoms were located in a difference Fourier map and refined freely. The remaining $\mathrm{H}$ atoms were positioned geometrically $[\mathrm{C}-\mathrm{H}=0.93-0.97 \AA]$ and refined using a riding model, with $U_{\text {iso }}(\mathrm{H})=1.2 U_{\text {eq }}(\mathrm{C})$ and $1.5 U_{\text {eq }}($ methyl C). A rotating group model was used for the methyl groups. 




\section{Figure 1}

The molecular structure of the title compound, with atom labels and 50\% probability ellipsoids for non-H atoms.

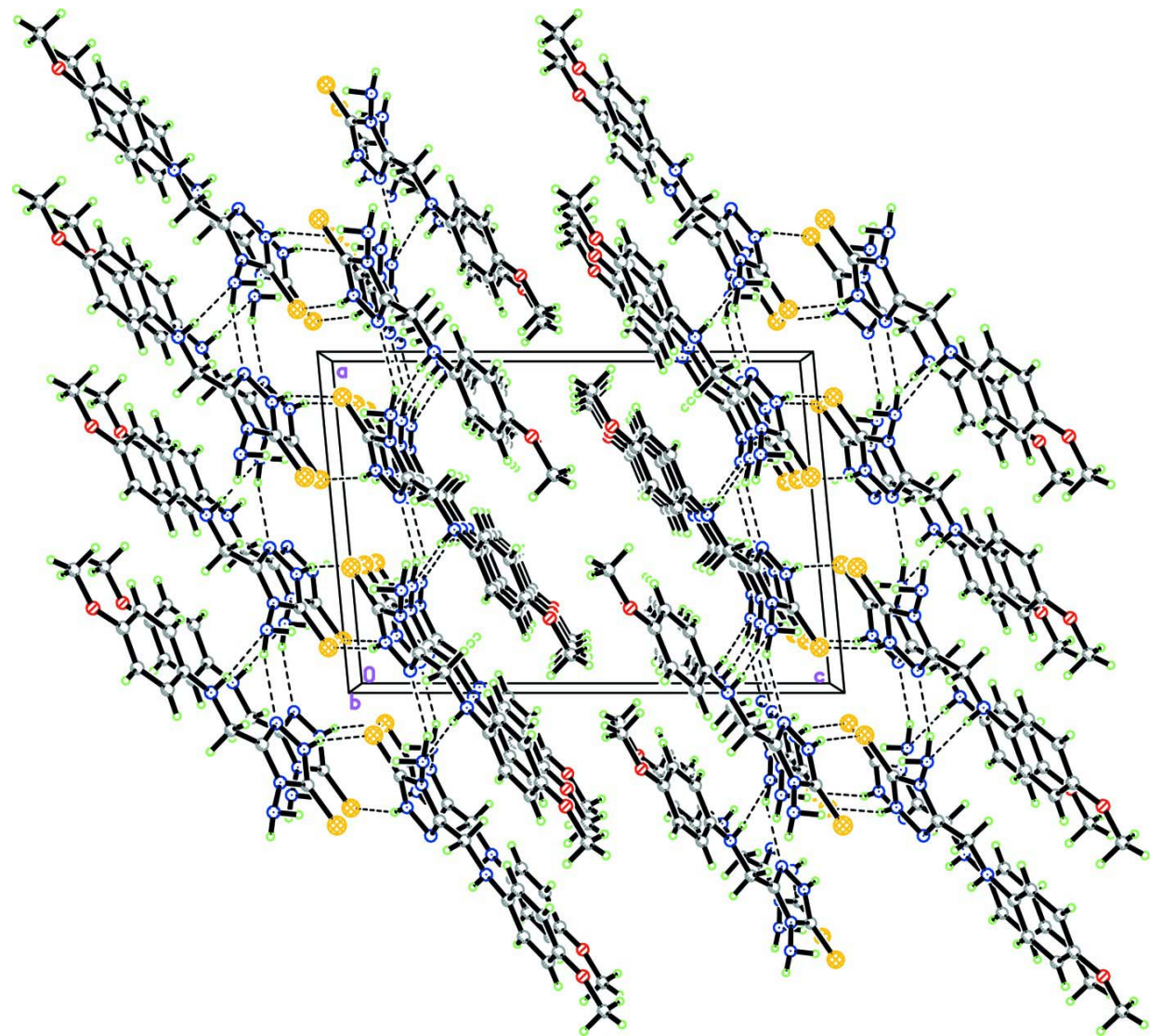

\section{Figure 2}

The crystal structure of the title compound, viewed down the $b$ axis, showing two-dimensional networks parallel to $a b$ plane. Intermolecular hydrogen bonds are shown as dashed lines.

\section{4-Amino-3-[(4-methoxyphenyl)aminomethyl]-1H-1,2,4-triazole- 5(4H)-thione}

\section{Crystal data}

$\mathrm{C}_{10} \mathrm{H}_{13} \mathrm{~N}_{5} \mathrm{OS}$

$M_{r}=251.31$

Monoclinic, $C 2$

Hall symbol: C 2y

$$
\begin{aligned}
& a=11.5142(2) \AA \\
& b=5.8804(1) \AA \\
& c=16.6891(3) \AA \\
& \beta=95.292(1)^{\circ}
\end{aligned}
$$


$V=1125.17(3) \AA^{3}$
$Z=4$
$F(000)=528$
$D_{\mathrm{x}}=1.484 \mathrm{Mg} \mathrm{m}^{-3}$

Mo $K \alpha$ radiation, $\lambda=0.71073 \AA$

Cell parameters from 2961 reflections

\section{Data collection}

Bruker SMART APEXII CCD area-detector diffractometer

Radiation source: fine-focus sealed tube

Graphite monochromator

$\varphi$ and $\omega$ scans

Absorption correction: multi-scan

(SADABS; Bruker, 2005)

$T_{\min }=0.913, T_{\max }=0.994$

\section{Refinement}

Refinement on $F^{2}$

Least-squares matrix: full

$R\left[F^{2}>2 \sigma\left(F^{2}\right)\right]=0.031$

$w R\left(F^{2}\right)=0.068$

$S=1.04$

2365 reflections

171 parameters

1 restraint

Primary atom site location: structure-invariant direct methods

Secondary atom site location: difference Fourier map $\theta=3.6-32.2^{\circ}$

$\mu=0.28 \mathrm{~mm}^{-1}$

$T=100 \mathrm{~K}$

Plate, colourless

$0.33 \times 0.18 \times 0.02 \mathrm{~mm}$

5827 measured reflections

2365 independent reflections

2126 reflections with $I>2 \sigma(I)$

$R_{\text {int }}=0.024$

$\theta_{\max }=30.0^{\circ}, \theta_{\min }=3.6^{\circ}$

$h=-15 \rightarrow 16$

$k=-8 \rightarrow 5$

$l=-23 \rightarrow 23$

Hydrogen site location: inferred from neighbouring sites

$\mathrm{H}$ atoms treated by a mixture of independent and constrained refinement

$w=1 /\left[\sigma^{2}\left(F_{\mathrm{o}}^{2}\right)+(0.0277 P)^{2}+0.6764 P\right]$ where $P=\left(F_{\mathrm{o}}^{2}+2 F_{\mathrm{c}}^{2}\right) / 3$

$(\Delta / \sigma)_{\max }=0.001$

$\Delta \rho_{\max }=0.33$ e $\AA^{-3}$

$\Delta \rho_{\min }=-0.26$ e $\AA^{-3}$

Absolute structure: Flack (1983), 588 Friedel pairs

Absolute structure parameter: 0.03 (7)

\section{Special details}

Experimental. The crystal was placed in the cold stream of an Oxford Cyrosystems Cobra open-flow nitrogen cryostat (Cosier \& Glazer, 1986) operating at 100.0 (1) K.

Geometry. All e.s.d.'s (except the e.s.d. in the dihedral angle between two 1.s. planes) are estimated using the full covariance matrix. The cell e.s.d.'s are taken into account individually in the estimation of e.s.d.'s in distances, angles and torsion angles; correlations between e.s.d.'s in cell parameters are only used when they are defined by crystal symmetry. An approximate (isotropic) treatment of cell e.s.d.'s is used for estimating e.s.d.'s involving 1.s. planes.

Refinement. Refinement of $F^{2}$ against ALL reflections. The weighted $R$-factor $w R$ and goodness of fit $S$ are based on $F^{2}$, conventional $R$-factors $R$ are based on $F$, with $F$ set to zero for negative $F^{2}$. The threshold expression of $F^{2}>\sigma\left(F^{2}\right)$ is used only for calculating $R$-factors $(\mathrm{gt})$ etc. and is not relevant to the choice of reflections for refinement. $R$-factors based on $F^{2}$ are statistically about twice as large as those based on $F$, and $R$-factors based on ALL data will be even larger.

Fractional atomic coordinates and isotropic or equivalent isotropic displacement parameters $\left(\AA^{2}\right)$

\begin{tabular}{lllll}
\hline & $x$ & $y$ & $z$ & $U_{\text {iso }} * / U_{\text {eq }}$ \\
\hline S1 & $0.37035(4)$ & $0.34412(9)$ & $0.03759(3)$ & $0.01571(11)$ \\
O1 & $-0.26796(11)$ & $1.2919(3)$ & $0.42305(8)$ & $0.0248(4)$ \\
N1 & $0.07536(13)$ & $0.3929(3)$ & $0.13658(9)$ & $0.0171(4)$ \\
N2 & $0.15394(14)$ & $0.2872(3)$ & $0.09067(10)$ & $0.0164(4)$ \\
N3 & $0.23563(13)$ & $0.5947(3)$ & $0.13167(9)$ & $0.0136(3)$ \\
N4 & $-0.02128(14)$ & $0.6826(3)$ & $0.24608(10)$ & $0.0167(4)$ \\
N5 & $0.30984(14)$ & $0.7845(3)$ & $0.14247(10)$ & $0.0171(4)$
\end{tabular}




$\begin{array}{lllll}\text { C1 } & 0.25184(15) & 0.4074(3) & 0.08580(11) & 0.0144(4) \\ \text { C2 } & 0.12844(15) & 0.5803(4) & 0.16083(11) & 0.0138(4) \\ \text { C3 } & 0.08129(16) & 0.7617(4) & 0.21027(12) & 0.0158(4) \\ \text { H3A } & 0.1404 & 0.8072 & 0.2524 & 0.019^{*} \\ \text { H3B } & 0.0613 & 0.8933 & 0.1768 & 0.019^{*} \\ \text { C4 } & -0.08548(14) & 0.8413(4) & 0.28707(10) & 0.0146(3) \\ \text { C5 } & -0.04122(16) & 1.0556(4) & 0.31036(12) & 0.0174(4) \\ \text { H5A } & 0.0307 & 1.1019 & 0.2950 & 0.021^{*} \\ \text { C6 } & -0.10392(16) & 1.1985(4) & 0.35610(12) & 0.0187(4) \\ \text { H6A } & -0.0732 & 1.3398 & 0.3715 & 0.022^{*} \\ \text { C7 } & -0.21184(16) & 1.1350(4) & 0.37941(11) & 0.0168(4) \\ \text { C8 } & -0.25711(16) & 0.9225(4) & 0.35578(11) & 0.0174(4) \\ \text { H8A } & -0.3295 & 0.8776 & 0.3708 & 0.021^{*} \\ \text { C9 } & -0.19487(15) & 0.7792(4) & 0.31039(11) & 0.0163(4) \\ \text { H9A } & -0.2261 & 0.6384 & 0.2949 & 0.020^{*} \\ \text { C10 } & -0.37125(17) & 1.2205(4) & 0.45705(12) & 0.0219(5) \\ \text { H10A } & -0.3982 & 1.3406 & 0.4896 & 0.033^{*} \\ \text { H10B } & -0.3545 & 1.0881 & 0.4897 & 0.033^{*} \\ \text { H10C } & -0.4305 & 1.1848 & 0.4147 & 0.033^{*} \\ \text { H1N2 } & 0.1335(18) & 0.175(4) & 0.0617(13) & 0.016(6)^{*} \\ \text { H1N4 } & -0.063(2) & 0.601(5) & 0.2156(15) & 0.032(7)^{*} \\ \text { H1N5 } & 0.381(2) & 0.728(5) & 0.1550(16) & 0.047(8)^{*} \\ \text { H2N5 } & 0.3141(19) & 0.845(6) & 0.0921(15) & 0.042(7)^{*} \\ \end{array}$

Atomic displacement parameters $\left(\AA^{2}\right)$

\begin{tabular}{lllllll}
\hline & $U^{11}$ & $U^{22}$ & $U^{33}$ & $U^{12}$ & $U^{13}$ & $U^{23}$ \\
\hline S1 & $0.01344(18)$ & $0.0157(2)$ & $0.0184(2)$ & $0.0019(2)$ & $0.00344(15)$ & $-0.0002(2)$ \\
O1 & $0.0220(7)$ & $0.0201(9)$ & $0.0342(8)$ & $-0.0015(6)$ & $0.0123(6)$ & $-0.0096(7)$ \\
N1 & $0.0157(7)$ & $0.0161(10)$ & $0.0201(8)$ & $-0.0007(7)$ & $0.0052(6)$ & $0.0000(7)$ \\
N2 & $0.0186(7)$ & $0.0117(9)$ & $0.0197(8)$ & $-0.0021(6)$ & $0.0050(6)$ & $-0.0030(7)$ \\
N3 & $0.0126(7)$ & $0.0149(9)$ & $0.0135(7)$ & $-0.0008(6)$ & $0.0015(6)$ & $-0.0003(7)$ \\
N4 & $0.0150(8)$ & $0.0149(9)$ & $0.0208(9)$ & $-0.0039(7)$ & $0.0051(7)$ & $-0.0033(7)$ \\
N5 & $0.0142(7)$ & $0.0148(9)$ & $0.0220(9)$ & $-0.0050(6)$ & $0.0006(6)$ & $-0.0019(7)$ \\
C1 & $0.0143(8)$ & $0.0155(10)$ & $0.0135(8)$ & $0.0025(7)$ & $0.0011(7)$ & $0.0001(7)$ \\
C2 & $0.0132(8)$ & $0.0156(10)$ & $0.0126(9)$ & $0.0022(7)$ & $0.0015(7)$ & $0.0029(8)$ \\
C3 & $0.0151(8)$ & $0.0140(10)$ & $0.0183(9)$ & $-0.0012(7)$ & $0.0020(7)$ & $0.0006(8)$ \\
C4 & $0.0153(7)$ & $0.0154(9)$ & $0.0132(7)$ & $0.0032(9)$ & $0.0012(6)$ & $0.0006(10)$ \\
C5 & $0.0139(9)$ & $0.0176(11)$ & $0.0211(10)$ & $-0.0015(8)$ & $0.0043(7)$ & $0.0005(8)$ \\
C6 & $0.0188(9)$ & $0.0146(10)$ & $0.0228(10)$ & $-0.0027(8)$ & $0.0020(8)$ & $-0.0037(8)$ \\
C7 & $0.0174(9)$ & $0.0165(10)$ & $0.0167(9)$ & $0.0015(8)$ & $0.0030(7)$ & $-0.0005(8)$ \\
C8 & $0.0157(9)$ & $0.0184(10)$ & $0.0187(9)$ & $-0.0014(8)$ & $0.0044(7)$ & $0.0013(8)$ \\
C9 & $0.0163(8)$ & $0.0121(10)$ & $0.0203(9)$ & $-0.0024(7)$ & $0.0002(7)$ & $-0.0006(8)$ \\
C10 & $0.0177(9)$ & $0.0258(13)$ & $0.0228(10)$ & $0.0016(9)$ & $0.0057(8)$ & $-0.0032(9)$ \\
& & & & & &
\end{tabular}


Geometric parameters $\left(\AA,{ }^{\circ}\right)$

\begin{tabular}{|c|c|c|c|}
\hline $\mathrm{S} 1-\mathrm{C} 1$ & $1.6883(18)$ & $\mathrm{C} 3-\mathrm{H} 3 \mathrm{~A}$ & 0.97 \\
\hline $\mathrm{O} 1-\mathrm{C} 7$ & $1.373(2)$ & $\mathrm{C} 3-\mathrm{H} 3 \mathrm{~B}$ & 0.97 \\
\hline $\mathrm{O} 1-\mathrm{C} 10$ & $1.427(2)$ & $\mathrm{C} 4-\mathrm{C} 5$ & $1.401(3)$ \\
\hline $\mathrm{N} 1-\mathrm{C} 2$ & $1.306(3)$ & $\mathrm{C} 4-\mathrm{C} 9$ & $1.401(2)$ \\
\hline $\mathrm{N} 1-\mathrm{N} 2$ & $1.386(2)$ & $\mathrm{C} 5-\mathrm{C} 6$ & $1.383(3)$ \\
\hline $\mathrm{N} 2-\mathrm{C} 1$ & $1.339(2)$ & $\mathrm{C} 5-\mathrm{H} 5 \mathrm{~A}$ & 0.93 \\
\hline $\mathrm{N} 2-\mathrm{H} 1 \mathrm{~N} 2$ & $0.84(2)$ & $\mathrm{C} 6-\mathrm{C} 7$ & $1.387(3)$ \\
\hline $\mathrm{N} 3-\mathrm{C} 1$ & $1.364(3)$ & C6-H6A & 0.93 \\
\hline $\mathrm{N} 3-\mathrm{C} 2$ & $1.370(2)$ & $\mathrm{C} 7-\mathrm{C} 8$ & $1.397(3)$ \\
\hline N3-N5 & $1.407(2)$ & $\mathrm{C} 8-\mathrm{C} 9$ & $1.377(3)$ \\
\hline $\mathrm{N} 4-\mathrm{C} 4$ & $1.407(3)$ & $\mathrm{C} 8-\mathrm{H} 8 \mathrm{~A}$ & 0.93 \\
\hline $\mathrm{N} 4-\mathrm{C} 3$ & $1.448(2)$ & C9-H9A & 0.93 \\
\hline $\mathrm{N} 4-\mathrm{H} 1 \mathrm{~N} 4$ & $0.82(3)$ & $\mathrm{C} 10-\mathrm{H} 10 \mathrm{~A}$ & 0.96 \\
\hline $\mathrm{N} 5-\mathrm{H} 1 \mathrm{~N} 5$ & $0.89(3)$ & $\mathrm{C} 10-\mathrm{H} 10 \mathrm{~B}$ & 0.96 \\
\hline $\mathrm{N} 5-\mathrm{H} 2 \mathrm{~N} 5$ & $0.92(3)$ & $\mathrm{C} 10-\mathrm{H} 10 \mathrm{C}$ & 0.96 \\
\hline $\mathrm{C} 2-\mathrm{C} 3$ & $1.482(3)$ & & \\
\hline $\mathrm{C} 7-\mathrm{O} 1-\mathrm{C} 10$ & $117.60(17)$ & $\mathrm{H} 3 \mathrm{~A}-\mathrm{C} 3-\mathrm{H} 3 \mathrm{~B}$ & 108.1 \\
\hline $\mathrm{C} 2-\mathrm{N} 1-\mathrm{N} 2$ & $103.83(15)$ & $\mathrm{C} 5-\mathrm{C} 4-\mathrm{C} 9$ & $118.09(18)$ \\
\hline $\mathrm{C} 1-\mathrm{N} 2-\mathrm{N} 1$ & $113.14(16)$ & $\mathrm{C} 5-\mathrm{C} 4-\mathrm{N} 4$ & $122.53(16)$ \\
\hline $\mathrm{C} 1-\mathrm{N} 2-\mathrm{H} 1 \mathrm{~N} 2$ & $125.0(14)$ & $\mathrm{C} 9-\mathrm{C} 4-\mathrm{N} 4$ & $119.3(2)$ \\
\hline $\mathrm{N} 1-\mathrm{N} 2-\mathrm{H} 1 \mathrm{~N} 2$ & $120.6(14)$ & $\mathrm{C} 6-\mathrm{C} 5-\mathrm{C} 4$ & $120.33(17)$ \\
\hline $\mathrm{C} 1-\mathrm{N} 3-\mathrm{C} 2$ & $108.88(16)$ & $\mathrm{C} 6-\mathrm{C} 5-\mathrm{H} 5 \mathrm{~A}$ & 119.8 \\
\hline $\mathrm{C} 1-\mathrm{N} 3-\mathrm{N} 5$ & $126.84(15)$ & $\mathrm{C} 4-\mathrm{C} 5-\mathrm{H} 5 \mathrm{~A}$ & 119.8 \\
\hline $\mathrm{C} 2-\mathrm{N} 3-\mathrm{N} 5$ & $124.04(17)$ & $\mathrm{C} 5-\mathrm{C} 6-\mathrm{C} 7$ & $121.20(19)$ \\
\hline $\mathrm{C} 4-\mathrm{N} 4-\mathrm{C} 3$ & $118.22(18)$ & $\mathrm{C} 5-\mathrm{C} 6-\mathrm{H} 6 \mathrm{~A}$ & 119.4 \\
\hline $\mathrm{C} 4-\mathrm{N} 4-\mathrm{H} 1 \mathrm{~N} 4$ & $112.7(18)$ & $\mathrm{C} 7-\mathrm{C} 6-\mathrm{H} 6 \mathrm{~A}$ & 119.4 \\
\hline $\mathrm{C} 3-\mathrm{N} 4-\mathrm{H} 1 \mathrm{~N} 4$ & $112.5(17)$ & $\mathrm{O} 1-\mathrm{C} 7-\mathrm{C} 6$ & $116.46(18)$ \\
\hline $\mathrm{N} 3-\mathrm{N} 5-\mathrm{H} 1 \mathrm{~N} 5$ & $105.8(19)$ & $\mathrm{O} 1-\mathrm{C} 7-\mathrm{C} 8$ & $124.69(17)$ \\
\hline $\mathrm{N} 3-\mathrm{N} 5-\mathrm{H} 2 \mathrm{~N} 5$ & $105.9(18)$ & $\mathrm{C} 6-\mathrm{C} 7-\mathrm{C} 8$ & $118.83(18)$ \\
\hline $\mathrm{H} 1 \mathrm{~N} 5-\mathrm{N} 5-\mathrm{H} 2 \mathrm{~N} 5$ & $103(2)$ & $\mathrm{C} 9-\mathrm{C} 8-\mathrm{C} 7$ & $120.19(17)$ \\
\hline $\mathrm{N} 2-\mathrm{C} 1-\mathrm{N} 3$ & $103.45(15)$ & $\mathrm{C} 9-\mathrm{C} 8-\mathrm{H} 8 \mathrm{~A}$ & 119.9 \\
\hline $\mathrm{N} 2-\mathrm{C} 1-\mathrm{S} 1$ & $129.38(15)$ & $\mathrm{C} 7-\mathrm{C} 8-\mathrm{H} 8 \mathrm{~A}$ & 119.9 \\
\hline $\mathrm{N} 3-\mathrm{C} 1-\mathrm{S} 1$ & $127.15(15)$ & $\mathrm{C} 8-\mathrm{C} 9-\mathrm{C} 4$ & $121.4(2)$ \\
\hline $\mathrm{N} 1-\mathrm{C} 2-\mathrm{N} 3$ & $110.68(18)$ & $\mathrm{C} 8-\mathrm{C} 9-\mathrm{H} 9 \mathrm{~A}$ & 119.3 \\
\hline $\mathrm{N} 1-\mathrm{C} 2-\mathrm{C} 3$ & $126.50(17)$ & $\mathrm{C} 4-\mathrm{C} 9-\mathrm{H} 9 \mathrm{~A}$ & 119.3 \\
\hline $\mathrm{N} 3-\mathrm{C} 2-\mathrm{C} 3$ & $122.78(18)$ & $\mathrm{O} 1-\mathrm{C} 10-\mathrm{H} 10 \mathrm{~A}$ & 109.5 \\
\hline $\mathrm{N} 4-\mathrm{C} 3-\mathrm{C} 2$ & $110.69(17)$ & $\mathrm{O} 1-\mathrm{C} 10-\mathrm{H} 10 \mathrm{~B}$ & 109.5 \\
\hline $\mathrm{N} 4-\mathrm{C} 3-\mathrm{H} 3 \mathrm{~A}$ & 109.5 & $\mathrm{H} 10 \mathrm{~A}-\mathrm{C} 10-\mathrm{H} 10 \mathrm{~B}$ & 109.5 \\
\hline $\mathrm{C} 2-\mathrm{C} 3-\mathrm{H} 3 \mathrm{~A}$ & 109.5 & $\mathrm{O} 1-\mathrm{C} 10-\mathrm{H} 10 \mathrm{C}$ & 109.5 \\
\hline $\mathrm{N} 4-\mathrm{C} 3-\mathrm{H} 3 \mathrm{~B}$ & 109.5 & $\mathrm{H} 10 \mathrm{~A}-\mathrm{C} 10-\mathrm{H} 10 \mathrm{C}$ & 109.5 \\
\hline $\mathrm{C} 2-\mathrm{C} 3-\mathrm{H} 3 \mathrm{~B}$ & 109.5 & $\mathrm{H} 10 \mathrm{~B}-\mathrm{C} 10-\mathrm{H} 10 \mathrm{C}$ & 109.5 \\
\hline $\mathrm{C} 2-\mathrm{N} 1-\mathrm{N} 2-\mathrm{C} 1$ & $-0.9(2)$ & $\mathrm{N} 3-\mathrm{C} 2-\mathrm{C} 3-\mathrm{N} 4$ & $-168.35(17)$ \\
\hline $\mathrm{N} 1-\mathrm{N} 2-\mathrm{C} 1-\mathrm{N} 3$ & $1.1(2)$ & $\mathrm{C} 3-\mathrm{N} 4-\mathrm{C} 4-\mathrm{C} 5$ & $-14.9(3)$ \\
\hline $\mathrm{N} 1-\mathrm{N} 2-\mathrm{C} 1-\mathrm{S} 1$ & $179.40(14)$ & $\mathrm{C} 3-\mathrm{N} 4-\mathrm{C} 4-\mathrm{C} 9$ & $169.36(17)$ \\
\hline
\end{tabular}




$\begin{array}{llll}\mathrm{C} 2-\mathrm{N} 3-\mathrm{C} 1-\mathrm{N} 2 & -0.9(2) & \mathrm{C} 9-\mathrm{C} 4-\mathrm{C} 5-\mathrm{C} 6 & 1.0(3) \\ \mathrm{N} 5-\mathrm{N} 3-\mathrm{C} 1-\mathrm{N} 2 & -175.43(17) & \mathrm{N} 4-\mathrm{C} 4-\mathrm{C} 5-\mathrm{C} 6 & -174.84(19) \\ \mathrm{C} 2-\mathrm{N} 3-\mathrm{C} 1-\mathrm{S} 1 & -179.25(15) & \mathrm{C} 4-\mathrm{C} 5-\mathrm{C} 6-\mathrm{C} 7 & -0.5(3) \\ \mathrm{N} 5-\mathrm{N} 3-\mathrm{C} 1-\mathrm{S} 1 & 6.3(3) & \mathrm{C} 10-\mathrm{O} 1-\mathrm{C} 7-\mathrm{C} 6 & -171.73(18) \\ \mathrm{N} 2-\mathrm{N} 1-\mathrm{C} 2-\mathrm{N} 3 & 0.2(2) & \mathrm{C} 10-\mathrm{O} 1-\mathrm{C} 7-\mathrm{C} 8 & 10.0(3) \\ \mathrm{N} 2-\mathrm{N} 1-\mathrm{C} 2-\mathrm{C} 3 & 177.94(18) & \mathrm{C} 5-\mathrm{C} 6-\mathrm{C} 7-\mathrm{O} 1 & -178.46(18) \\ \mathrm{C} 1-\mathrm{N} 3-\mathrm{C} 2-\mathrm{N} 1 & 0.4(2) & \mathrm{C} 5-\mathrm{C} 6-\mathrm{C} 7-\mathrm{C} 8 & -0.1(3) \\ \mathrm{N} 5-\mathrm{N} 3-\mathrm{C} 2-\mathrm{N} 1 & 175.13(17) & \mathrm{O} 1-\mathrm{C} 7-\mathrm{C} 8-\mathrm{C} 9 & 178.47(18) \\ \mathrm{C} 1-\mathrm{N} 3-\mathrm{C} 2-\mathrm{C} 3 & -177.35(17) & \mathrm{C} 6-\mathrm{C} 7-\mathrm{C} 8-\mathrm{C} 9 & 0.2(3) \\ \mathrm{N} 5-\mathrm{N} 3-\mathrm{C} 2-\mathrm{C} 3 & -2.7(3) & \mathrm{C} 7-\mathrm{C} 8-\mathrm{C} 9-\mathrm{C} 4 & 0.2(3) \\ \mathrm{C} 4-\mathrm{N} 4-\mathrm{C} 3-\mathrm{C} 2 & -172.48(15) & \mathrm{C} 5-\mathrm{C} 4-\mathrm{C} 9-\mathrm{C} 8 & -0.8(3) \\ \mathrm{N} 1-\mathrm{C} 2-\mathrm{C} 3-\mathrm{N} 4 & 14.2(3) & \mathrm{N} 4-\mathrm{C} 4-\mathrm{C} 9-\mathrm{C} 8 & 175.13(18)\end{array}$

Hydrogen-bond geometry $\left(A,{ }^{\circ}\right)$

\begin{tabular}{lllll}
\hline$D-\mathrm{H} \cdots A$ & $D-\mathrm{H}$ & $\mathrm{H} \cdots A$ & $D \cdots A$ & $D-\mathrm{H} \cdots A$ \\
\hline $\mathrm{N} 2-\mathrm{H} 1 N 2 \cdots \mathrm{S} 1^{\mathrm{i}}$ & $0.84(2)$ & $2.55(2)$ & $3.3672(18)$ & $164(2)$ \\
$\mathrm{N} 4-\mathrm{H} 1 N 4 \cdots \mathrm{N} 5^{\mathrm{ii}}$ & $0.82(3)$ & $2.60(3)$ & $3.410(2)$ & $169(3)$ \\
$\mathrm{N} 5-\mathrm{H} 1 N 5 \cdots \mathrm{N} 1^{i i i}$ & $0.89(2)$ & $2.48(2)$ & $3.133(2)$ & $130(2)$ \\
\hline
\end{tabular}

Symmetry codes: (i) $-x+1 / 2, y-1 / 2,-z$; (ii) $x-1 / 2, y-1 / 2, z$; (iii) $x+1 / 2, y+1 / 2, z$. 